\author{
Marika Piva* \\ Università di Padova
}

\title{
IL SIGNOR DI CHATEAUBRIAND VOLTATO IN ITALIANO. MEDIAZIONI OTTOCENTESCHE
}

Abstract: A partire dal reperimento delle caratteristiche che permetterebbero la creazione di un repertorio ragionato delle traduzioni italiane di Chateaubriand e dalla presentazione di alcuni casi significativi, l'articolo si propone di sottolineare come lo studio della mediazione ottocentesca necessiti un approccio transdisciplinare. L'analisi di due raccolte di inizio Ottocento delle opere di Chateaubriand apre la riflessione sulla trasmissione della cultura d'oltralpe nel contesto italiano.

Parole chiave: Chateaubriand, storia della mediazione, pratica e teoria della traduzione, ricezione italiana della letteratura francese, cultura del XIX secolo, studi transdisciplinari

Si sostiene ch'è inutile tradurre libri dal francese perché tutti gli italiani sanno il francese.

Ma non è punto vero che tutti gli italiani sappiano il francese.

Molti poi, che più o meno esattamente lo capiscono a senso, ne storpiano la pronunzia a tal punto da perdere, leggendo un libro che abbia valore d'arte, gran parte del suo segreto valore; e s'immaginano di leggere la prosa di Chateaubriand come lo strimpellatore s'immagina di eseguire Chopin. Il buon traduttore li affranca da questo equivoco.

Giuseppe Antonio Borgese

Non esiste ancora un catalogo delle traduzioni italiane di Chateaubriand, primo passo imprescindibile per lo studio di una ricezione poco studiata se non per assaggi, spesso suggestivi, ma non necessariamente rappresentativi del contesto nel suo insieme. Partendo da un'analisi materiale dei volumi

\footnotetext{
*marika.piva@unipd.it
} 
dispersi, fin da inizio Ottocento, su tutta la penisola si evidenzia, infatti, una serie nutritissima di ristampe e contraffazioni che, da un lato, sottolineano la fortuna di alcune opere e, dall'altro, rendono meno immediato stabilire il reale numero delle traduzioni di ogni singolo testo. È proprio questo riciclo, ben noto agli studiosi del periodo, che viene ironicamente sottolineato nel 1840 in una telegrafica segnalazione della Rivista Viennese di quattro nuove pubblicazioni, di cui due del nostro autore (I Martiri o il trionfo della religione cristiana, di Chateaubriand, Milano, Tipografia di Paolo Lampato; Genio del cristianesimo, di Chateaubriand, Versione di Luigi Toccagni, illustrata con circa 700 intagli, Torino dallo stabilimento tipografico Fontana), unite a un'edizione illustrata di Abelardo ed Eloisa e una di Paolo e Virginia:

Anche queste quattro opere sono riprodotte in italiano coi politipi delle edizioni francesi, ond'è che potrebbe taluno per avventura inferirne che i librai della capitale lombarda, in mezzo a questo lusso di edizioni illustrate, altro non facciano alla finfine che vestire traduzioni per lo più già stampate, di ornamenti usati, abbandonati loro dai proprietarii, come vediamo i principi e le duchesse da tragedia vestir la porpora sbiadita e l'ermellino roso dai tarli, che ormai troppo disdirebbe alle corti (Bolza 1840: 289-290).

La pointe serve a introdurre - nell'ultima sezione della «Rassegna bibliografica», dedicata alle opere illustrate - un'edizione della Gerusalemme liberata destinata a risollevare l'onore nazionale superando «le più belle edizioni figurate, che ci siano finora venute di Francia» (Bolza 1840: 290). La proliferazione a metà secolo di testi tradotti in italiano accompagnati da illustrazioni sembrerebbe, insomma, non far altro che riproporre versioni e immagini già viste sancendo una ricezione acritica di oggetti esteticamente pregevoli ed evidentemente di grande successo.

Se una lista di titoli e date delle traduzioni di Chateaubriand apparse in Italia apporterebbe dunque poco, più utile pare abbozzare una tassonomia descrittiva basata su una prima ricognizione che si propone, al contempo, di controllare la versione del testo tradotto - com'è ben noto alcune opere sono oggetto di riedizioni in cui l'autore stesso corregge il testo e, soprattutto, interviene sul paratesto -, il contenuto dei tomi - i titoli dei volumi spesso si limitano al testo posto in apertura che però, non di rado, è seguito da altri -, nonché la presenza o meno di paratesti del traduttore o dell'editore, fonte incommensurabile di informazioni nonché, talvolta, di letture che influenzano non solo la ricezione, ma la vita dell'autore stesso. Mi limito a questo proposito a segnalare come, nei Mémoires d'outre-tombe, Chateaubriand (2003-2004, 1: 834) sottolinei 
che la prefazione della traduzione inglese dei $\operatorname{Martyrs}^{1}$, rendendo evidenti le allusioni alla politica imperiale, abbia contribuito al peggioramento dei suoi rapporti con Napoleone.

Un ulteriore passo avanti nella ricerca consiste nel reperimento di segnalazioni e recensioni contenenti traduzioni parziali all'interno di riviste e periodici, rassegna destinata a non raggiungere mai l'esaustività, ma che apporta dettagli essenziali al completamento del quadro d'insieme. A titolo di esempio, è utile evidenziare come estratti dei testi di Chateaubriand in italiano siano presenti anche in opere consacrate ad autori inglesi: il Teatro completo di Shakespeare di Carlo Rusconi (1838) e il Teatro di Shakespeare scelto e tradotto in italiano di Giulio Carcano (1845) utilizzano come introduzione alle traduzioni italiane del drammaturgo inglese la medesima versione di un capitolo dell'Essai sur la littérature anglaise (1836) del letterato francese, la cui uscita ha una certa eco nella penisola. Per limitarci all'anno di pubblicazione, quest'opera viene segnalata nel Ricoglitore italiano e straniero (III/II a nome di Cesare Cantù), nella Biblioteca Italiana (LXXXIV), nel Propagatore Religioso (I, 21), nella Gazzetta ufficiale (X), nonché nell'Indicatore. Raccolta periodica di articolo scelti (IV) che propone la traduzione della parte dedicata a Lutero.

Un'attenzione particolare meritano i testi su Milton e le sue traduzioni in Italia. Andrea Maffei fa precedere alla propria versione del Paradiso Perduto, pubblicato nel 1857, due pagine che si aprono con la seguente affermazione:

Di tanti che scrissero intorno al Paradiso Perduto, nessuno, a parer mio, c'innamora e c'invoglia a leggerlo e meditarlo, come il Chateaubriand nel Saggio sulla letteratura inglese. Critico profondo ed eccellente poeta egli stesso, ne svolge con tanta grazia ed evidenza le innumerevoli bellezze, che stimai né disutile, né inopportuno di premettere alla mia traduzione le cose che egli ne disse, e così risparmiare ai lettori una tediosa ripetizione di quanto fu detto e ridetto dall'Addison fino a noi (Maffei \& gli editori 1857: xiii).

Solo successivamente Maffei passa a considerazioni sul proprio metodo traduttivo, breve disamina in cui non si fa alcun accenno alle oggi celebri affermazioni sulla traduzione a opera di Chateaubriand, né si fa parola del fatto che i due volumi dell'Essai siano serviti da introduzione alla versione

${ }^{1}$ Si tratta di The Martyrs; or, The Triumph of the Christian Religion, a opera del cattolico William Joseph Walter, pubblicata nel 1812 per J. Ebers a Londra e oggetto di una seconda edizione nel 1819 con titolo The Two Martyrs. Nella «Translator's Preface» ci si auspica un'unione facente capo a Roma che si scagli contro il secondo Attila per distruggere l'impero; cfr. la nota 15 di Jean-Claude Berchet a Chateaubriand 2003-2004, 1: $1471-1472$. 
in prosa che il letterato francese ha fatto del poema di Milton ${ }^{2}$. Non è questa la sede per un confronto tra le due traduzioni che si fanno portavoce di un'appassionata riscoperta di questo testo nel corso dell'Ottocento e in cui scelte stilistiche e motivazioni ideologiche sono strettamente interdipendenti. L'aspetto che mi preme sottolineare è come, tanto Chateaubriand prima quanto Maffei dopo, evidenzino i legami tra grandi figure e la loro fedeltà al testo che prende forme differenti, ma assicura in entrambi i casi una comunione tra il traduttore e l'autore del testo originale. Colpisce come il poeta italiano definisca il letterato francese «critico profondo ed eccellente poeta egli stesso», ma eviti il confronto sull'ambito traduttivo - elemento che porta a interrogarsi sulla reale conoscenza in Italia della versione francese di Milton a opera di Chateaubriand e soprattutto sulla diffusione e la fortuna della concezione della traduzione esplicitata tanto nell' «Avertissement» che precede 1'Essai quanto nelle «Remarques» poste in apertura del Paradis Perdu, «Remarques» che vengono riprese nella già citata recensione apparsa nella Biblioteca Italiana. Per tornare al volume del 1857, le 71 pagine tratte dall'Essai, utile ricordarlo, non vengono tradotte in italiano, ma sono proposte nella versione originale che contiene al suo interno numerose citazioni in inglese, creando quindi un curioso plurilinguismo. Gli editori della traduzione italiana in versi di Milton, simmetricamente a quanto fatto dal traduttore, avevano chiuso la premessa che precede il paratesto di Maffei con un paragrafo volto a proporre l'autore francese esclusivamente come un «grande letterato» che presenta e giudica il «genio inglese» e non come uno dei suoi traduttori:

In fine volendo presentare qualche notizia sulla vita del Milton, sulle opere di lui e sulle vicende e tendenze de' suoi tempi, per non restar indietro a quanto ne scrisse con bella luce di critica e di storia quel sovrano ingegno del Chateaubriand, riproduciamo il brano de' suoi studi sulla letteratura d'Inghilterra, che si riferisce appunto al nostro poeta, nella certezza, che il lettore ci saprà grado trovando in questo libro illustrato e giudicato il lavoro del genio inglese colle proprie parole del grande letterato di Francia (Maffei \& gli editori 1857: vii).

Anche in questo caso, come accade nella segnalazione della Rivista viennese del 1840, il finale è rivolto ai connazionali a sottolineare - qui in un'ottica evidentemente commerciale e pubblicitaria - come le edizioni italiane siano al passo con i tempi: «E così ci continuino i nostri connazionali quel favore, che noi ci studiamo di ognor più meritare con le nostre diverse e pur sempre utili imprese» (ibidem). Vi si potrebbe intravedere un'eco di quella polemica iniziata nel gennaio del 1816 con la pubblicazione, nel primo numero della Biblioteca Italiana, della traduzione del saggio di Madame de

\footnotetext{
${ }^{2}$ Per una panoramica su Chateaubriand traduttore si veda Bougeard-Vetö (2005).
} 
Staël, Sulla maniera e la utilità delle traduzioni, che invitava i letterati della penisola a tradurre le opere inglesi e tedesche presentando la mediazione linguistica come un principio di perfettibilità, arricchimento e dinamismo (vedi, tra i molti, Wilhelm 2004).

Questo disinteresse italiano nei confronti della versione del Paradis perdu di Chateaubriand sembra rappresentare la situazione critica in senso più ampio: gli studi sull'autore si rivelano infatti particolarmente frammentari e discontinui rispetto alla storia della traduzione, intesa tanto come storia delle opere tradotte quanto come storia dei metodi di traduzione e del pensiero traduttologico. Quello che mi pare interessante indagare, anche e soprattutto in connessione al letterato francese, è invece proprio la traduzione come elemento della storia della cultura e della storia del processo traduttivo stesso $^{3}$, aspetto per il quale non risulta del tutto peregrino gettare uno sguardo sull'atteggiamento di Chateaubriand nei confronti di alcuni elementi connessi alla mediazione.

Com'è noto, l'attività di traduzione è presente e a tratti centrale nella vita e nella produzione dell'autore, che non manca di incontrare e citare nelle sue opere i propri traduttori. Il quinto capitolo del Libro XXXIX dei Mémoires d'outre-tombe, datato "Venise, septembre 1833», si apre con l'affermazione «À Venise, en 1806, il y avait un jeune signor Armani, traducteur italien ou ami du traducteur du Génie du Christianisme» (Chateaubriand 2003-2004, 2: 833); è in realtà la moglie di Chateaubriand, nel suo Cahier rouge, a offrire qualche dettaglio in più su Giovan Battista Armani (1768-1815), autore di tragedie e poeta, nonché appunto traduttore della versione del Génie pubblicata a Venezia nel 1805 e successivamente dei Martyrs (1814). Chateaubriand, nel 1833 già impegnato nella colossale traduzione di Milton, fa prova di un disinteresse di massima nei confronti di chi aveva tradotto due delle sue opere, al punto da non preoccuparsi del fatto che Armani fosse il reale traduttore o semplicemente un amico di questi. A Conegliano, similmente, l'autore francese riceve i complimenti degli amici di quella che definisce «une dame, traducteur de l'Abencérage» (Chateaubriand 2003-2004, 2: 909) - si tratta di Edvige de' Battisti di San Giorgio de Solari (1808-1867) che gli aveva inviato i suoi versi Lamento dell'ultimo Abencerraggio (cfr. Fedelini 1932) ${ }^{4}$ - e nei confronti

${ }^{3}$ In linea con la metodologia tracciata da Gideon Toury $(1980,1995)$ e l'idea di traduzione totale così come espressa da Peeter Torop (2009).

${ }^{4} \mathrm{Vi}$ sono riprodotti in fac-simile la busta e il testo della lettera di risposta di Chateaubriand datata «Paris, 11 avril 1833» («Vous avez, Madame, harmonieusement exprimé les regrets de mon Abencerage dans la langue de Pétrarque et de Tasse. La bienveillance de votre Muse augmente mes regrets d'avoir quitté cette belle Italie que j'ai tant aimée et que je ne reverrai peut-être plus») e i 128 versi del Lamento. 
della quale il memorialista dichiara di non essere più in debito dopo aver citato l'estratto della descrizione della protagonista del proprio racconto, Blanca, ritratto che, a detta dell'autore, sicuramente corrisponde a quello della traduttrice delle sue fantasticherie spagnole. Che il fascino femminile si affianchi, nei pensieri di Chateaubriand, alle considerazioni sulla diffusione delle sue opere è già evidente a Ferrara, dove la propria fama viene evocata con queste parole: «Pour la vieille Italie, je suis le défenseur de la religion; pour la jeune, le défenseur de la liberté; pour les autorités, j'ai l'honneur d'être la Sua Eccellenza GIA ambasciadore di Francia à Vérone et à Rome. Des dames, toutes sans doute d'une rare beauté, ont prêté la langue d'Angélique et d'Aquilan le Noir à la Floridienne Atala et au More Aben-Hamet» (Chateaubriand 2003-2004, 2: 882). Il problema della fedeltà delle versioni e della loro diffusione appare quanto mai lontano dalle preoccupazioni del memorialista, che si fregia però - come è logico che sia - dell'ampiezza della sua reputazione sottolineando, en passant, come il mestiere del traduttore fosse all'epoca spesso appannaggio delle donne, destinate a rimanere una folla anonima, degna tutt'al più di essere citata nel suo complesso o con il generico appellativo «une dame, traducteur». La bellezza evocata è quella femminile, senza alcun accenno a quella presente - o assente - nelle versioni italiane che, d'altro canto, evidentemente non erano mai state nemmeno sfogliate dall'autore.

In breve, la ricerca sulla mediazione di Chateaubriand appare come aperta e per ora si limita a interventi sporadici e asistematici mentre necessita, invece, di analisi che mettano in contatto aspetti diversi. Quello che propongo in queste pagine è una sorta di attraversamento per campioni significativi, volto a segnalare una serie di piste di indagine e non certo a tracciare un quadro sistematico. Emerge così come sia quanto mai imprescindibile l'incrocio di competenze diverse che permettano di evitare la separazione di approcci bibliometrici, archivistici, storico-culturali, letterari, stilistici, linguistici... dato che solo una reale transdisciplinarità può portare a cogliere le interazioni e le reciprocità che rendono le mediazioni culturali il vero specchio di un'epoca. Proprio in quest'ottica, non offro qui alcuna incursione nelle traduzioni propriamente dette: un'analisi sincronica e diacronica dei testi mi pare debba essere preventivamente inserita in una panoramica più ampia per poter rivelare quanto le versioni riflettano i modelli culturali, il contesto letterario, le teorie sulla traduzione e la consapevolezza del traduttore del suo ruolo.

La storia delle traduzioni italiane di Chateaubriand è strettamente legata all'evoluzione della ricezione dell'autore nella penisola di cui offre una sintesi Ivanna Rosi (2015), basandosi principalmente sugli studi di Rabiz- 
zani (1918) e Cordié (1969) . L'immediata e ampia ripercussione di parte delle opere dell'Enchanteur riguarda vari ambiti della sua ricca e variegata produzione, da cui rimangono esclusi a lungo i Mémoires d'outre-tombe (1848-1849), opera postuma e sorta di summa: come sottolinea Rosi, ne vengono per la prima volta riuniti alcuni stralci dallo stesso Rabizzani nel 1910 in Viaggio in Italia, aggiuntevi pagine dei Martiri e delle Memorie d'oltre-tomb $a^{6}$, mentre la prima traduzione italiana integrale è quella progettata da Garboli e uscita nel 1995 nella Biblioteca della Pléiade/Einaudi. Sorte ancora più avversa in Italia hanno la prima opera di Chateaubriand, l'Essai historique sur les révolutions (1797), pubblicata nel 2006 nella traduzione di Edi Pasini, e l'Essai sur la littérature anglaise la cui prima versione italiana appare solo nel 2010 a cura dalla scrivente. Molte, d'altro canto, le opere di grande e immediato successo di cui viene intrapresa rapidamente la traduzione, che la studiosa divide in tre settori piuttosto eterogenei: la parte narrativo-epica-apologetica composta da Natchez, Abencérage, Génie e Rancé; gli scritti di viaggio; la produzione politica a cui viene legata anche quella storica.

Ogni testo, come è ovvio, ha una storia a sé; basti qui citare quello che probabilmente rappresenta il successo più ampio e immediato dell'autore francese, Atala, episodio staccato dal Génie du Christianisme e pubblicato nel 1800. Nell' 'Avertissement» dell' edizione francese in $18^{\circ}$ pubblicata a Lyon nel 1804 - segnalata come «Quatrième édition» nel frontespizio -, gli editori (Ballanche père et fils) sottolineano l'enorme fortuna del Génie, pubblicato in aprile del 1802 e già in luglio oggetto di una contraffazione ad Avignone di cui parla lo stesso Chateaubriand nei Mémoires (2003-2004, 1: 657). Dopo aver tracciato il catalogo delle edizioni - e la spiegazione dettagliata tanto delle particolarità di ognuna, quanto della ragione alla base del-

${ }^{5}$ Rosi si occupa della ricezione tra il 1834 e il 1994, la tranche successiva, dal 1995 al 2015, è a opera di Fabio Vasarri (2015).

${ }^{6}$ Similmente, nel 1950, Carlo Pellegrini riunisce frammenti di vari scritti dell'autore nel volume Chateaubriand pubblicato da Garzanti. D'altro canto escono parti estrapolate dai Mémoires e concepite come autonome: già nel 1850 appare, anonima, la Storia di Napoleone narrata da Chateaubriand nelle sue Memorie l'oltre tomba per Marietti (i libri su Napoleone vengono poi pubblicati nel 1969 da Sansoni a opera di Orsola Nemi, con saggio introduttivo di Giovanni Macchia), mentre nel 1959 Mario Picchi traduce solo il Congedo dal mondo per Morcelliana. È nel 1923 che Giuseppe Gallavresi crea la prima antologia italiana dell'opera per la sua «Collezione di Memorie» pubblicata da Facchi, seguito da Vitalino Brancati nel 1942 per «Il sofà delle muse» di Rizzoli (ristampe del 1943 e 1945, ripreso da Longanesi nel 1973 e nel 1983). Nel 1950 Glauco Natoli pubblica un'ulteriore scelta di Mémoires per D'Anna dando ampio spazio ai primi 12 libri (ristampa del 1957) e nel 1959 esce l'ampia traduzione commentata in due volumi di Eva Timbaldi Abruzzese per Utet (ristampa del 1968). Cfr. Cordié (1969) e Rosi (2015). 
l'apparentemente insensato conteggio delle edizioni -, nell' «Avertissement» si passa a una rapida recensione della ricezione europea e si dichiara che, per non lasciare nulla a desiderare al lettore, si era prospettata una lista esatta delle traduzioni di Atala e del Génie du Christianisme, ma che si è costretti a limitarsi a quelle di cui è giunta notizia in Francia. La parte relativa all'italiano annovera quattro traduzioni del racconto americano, elencate con un inspiegabile inversione cronologica: la prima di Blanvillain, traduttore di Paul et Virginie, pubblicata a Parigi nel 1801; la seconda a opera dell'abate L.I.T., pubblicata a Venezia nel 1803; la terza di P. L. Constantini, dedicata alla duchessa di York e d'Albany, pubblicata a Berlino nel 1802; la quarta apparsa nell'inverno 1804 per Piatti, a Firenze, prima di una raccolta di traduzioni di romanzi. Quanto al Génie, la sua traduzione è opera del dottor Rosini che ne ha fatto pubblicare i primi due volumi a Pisa per la Società letteraria; iniziata nel 1802, nel 1804 l'impresa è ancora in corso. Appare evidente come la descrizione della fortuna editoriale del testo in patria e della sua ampia ricezione all'estero serva a giustificare l'ennesima edizione in Francia e a spingere il pubblico ad acquistarla, azioni che anche gli editori e i traduttori italiani si troveranno a svolgere. Questo paratesto francese apre su varie piste ancora tutte da indagare:

- la pubblicazione di traduzioni italiane fuori d'Italia, nello specifico a Parigi e Berlino;

- la segnalazione o meno del nome dei traduttori sul frontespizio e la loro identificazione - la figura di Blanvillain, nato a Orléans verso il 1758, è stata oggetto di alcune ricerche legate a Chateaubriand (vedi Sullam Calimani 1998), mentre 1'abate L.I.T., che appare come il traduttore di Atala già nell'edizione veneziana per Francesco Andreola del 1802, si rivela essere il gesuita svedese Lorenzo Ignazio Thjulen ed esula dalle ricerche specifiche sull'autore francese, così come la sua attività di traduttore non viene sottolineata dagli studi che gli sono dedicati (cfr. Guerra 2004)7;

- $\quad$ il ruolo svolto da alcuni librai, nello specifico Guglielmo Piatti ${ }^{8}$; è in effetti a Firenze che Atala viene posta in testa alla Raccolta di romanzi tradotti da varie lingue, in cinque volumi, che vedono

${ }^{7}$ Il trait-d'union è stato fornito da Bruno Berni, il cui suggerimento ha permesso di condurre in porto quella che, per utilizzare le parole di Berman (1995), si potrebbe letteralmente definire una «recherche du traducteur».

${ }^{8}$ Berchet (2012), nella sua biografia, segnala come il primo soggiorno italiano di Chateaubriand come segretario di ambasciata a Roma implichi anche un impegno nella diffusione e traduzione della propria opera. Nello specifico, il passaggio per Firenze lo porta a stringere contatti proprio con il libraio Guglielmo Piatti che si dimostrerà un intermediario efficace. Cfr. Riberette (1965-1966). 
come seconda uscita René, seguito da Margaretta contessa di Rainsford ossia L'interno d'una famiglia traduzione dall'inglese (2 vol.) e La Galatea romanzo pastorale già tirato dallo spagnuolo di Michele Cervantes dal signore di Florian tradotto in italiano;

- le scelte editoriali, in particolare la collocazione delle opere in sillogi miscellanee o in raccolte dedicate all'autore.

In questa sede mi limito ad attirare l'attenzione sulle raccolte ottocentesche di Chateaubriand, due a mia conoscenza in Italia (cfr. Appendice).

Le Opere Varie del visconte Chateaubriand recate in italiano, pubblicate in 24 volumi $^{10}$ da Girolamo Tasso a Venezia tra il 1827 e il 1830 , sono la prima traccia della fortuna delle Euvres complètes (1826-1831) progettate dall'autore, che causeranno il fallimento dell'editore Ladvocat e che non risponderanno appieno alle aspettative che lo scrittore vi poneva, ma che saranno nondimeno immediatamente recepite in Italia come prova, appunto, questa impresa veneziana.

Il primo volume edito da Tasso si apre con Avventure dell'ultimo Abencerragio, «Prima traduzione italiana», come l'editore si cura di sottolineare nelle pagine immediatamente seguenti: «Mi giova sperare che i lettori accoglieranno favorevolmente l'offerta ch'io fo loro di questo libro, che per la prima volta vede la luce sotto veste italiana» (AA.VV. 1827-30, 1: n.p. $[8])^{11}$. Il secondo e il terzo volume di questa raccolta contengono I Natchi, poema, a sua volta «Prima traduzione italiana»; in questo caso l'opera è preceduta da un brevissimo testo indirizzato «Ai lettori» dove si dichiara

${ }^{9}$ Quanto agli ultimi due titoli della raccolta fiorentina, si tratta in entrambi i casi di traduzioni di traduzioni: la prima è quella di Alexandrine-Louise Boutinon des Hayes de Courcelles (1758-1826), pubblicata nel 1797 e che è la traduzione dell'anonimo Margaretta, Countess of Rainsford. A Sentimental Novel risalente al 1769. Tanto il romanzo inglese quanto quello spagnolo giungono in Italia tramite le versioni francesi.

${ }^{10}$ E non 18 come segnalato da Rosi, che pure recensisce questa raccolta «sfuggita a Cordié» (2015: clx). D'altro canto quest'ultimo, in apertura del suo intervento, dichiara esplicitamente di presentare alcune considerazioni basate su una ricerca incompleta che si limita alle opere presenti nella biblioteca Nazionale Centrale di Firenze, nel Gabinetto scientifico-letterario Vieusseux e nella biblioteca Marucelliana.

${ }^{11}$ L'Editore si riserva le pagine 7 e 8, non numerate e che precedono immediatamente il testo, riassumendo di fatto quanto dichiarato da Chateaubriand stesso nella propria prefazione al racconto; il volume contiene di seguito le Considerazioni su l'Inghilterra e su gl'inglesi, le Considerazioni sopra Young e le Considerazioni sopra Shakspeare. Non si intende evidentemente rispettare le divisioni dell'edizione Ladvocat, visto che il primo volume pubblicato in Francia - il sedicesimo della raccolta - proponeva i tre racconti Atala, René e l'Abencérage, mentre in questa edizione italiana i due testi più noti vengono raccolti nel sesto volume. 
che «il traduttore ha creduto di attenersi il più che potè al linguaggio messo in bocca ai selvaggi dal Signor Chateaubriand» (AA.VV. 1827-30, 2: n.p. [5]) ed è seguita da un «Avviso ai signori associati» volto a specificare la duplice natura dell'opera, nella prima parte poema e nella seconda parte «continuazione» dove la narrazione appunto continua, ma vi è un netto scarto stilistico. Al fine di rendere la comprensione della storia più agevole a chi non conosca le vicende di René, si decide di anticipare la pubblicazione del sesto volume, contenente il racconto omonimo: «siccome però quest'operetta è troppo scarsa alla mole conveniente ad un volume, ci aggiungerò l'Atala, altro racconto che strettamente si lega alla storia di Chactas e dei Natchi, e della quale non pochi lettori avranno sentito il desiderio, leggendo i Natchi suddetti» (AA.VV. 1827-30, 3: n.p. [179]).

L'editore italiano stravolge l'ordine di uscita dei testi al punto da interrompere un'opera per pubblicarne prima altre due riunite in un medesimo volume; così facendo di fatto preserva la politica di base dell'edizione Ladvocat: creare dei volumi non eccessivamente smilzi e offrire al lettore testi inediti intervallandoli ad altri ben noti. La scelta editoriale si fa anche carico della minore conoscenza dei personaggi da parte del pubblico della penisola e si premura di rendere la fruizione delle vicende narrate più agevole possibile. Nel quarto volume delle Opere varie, contenente appunto I Natchi. Continuazione, il testo è preceduto da un «Avvertimento del traduttore» che specifica nuovamente come i due volumi trattino la medesima materia dei due precedenti, ma come siano stati scritti con un altro tenore: l'autore, «smettendo ora il carattere di poeta, assume quello di semplice storico, e per conseguenza la narrazione procede con più rapidità da un canto, e con minor corredo di adornamenti dall'altro. Era necessario che i lettori fossero di ciò avvertiti, acciocchè non credessero che il traduttore alterasse a suo senno l'indole della scrittura francese, quand'egli anzi si studia di attenersi, per quanto possibile, ad essa scrupolosamente» (AA.VV. 1827-30, 4: n.p. $[5])^{12}$. Gli interventi del traduttore sono tutti volti a prevenire eventuali critiche relative allo stile: l'attenersi al testo di partenza, soprattutto quando questo è poco noto, diseguale e di molto diverso rispetto a quello utilizzato nelle altre opere dell'autore, rischia di esporre a giudizi severi.

Alla fine del decimo volume, contenente il Viaggio in Italia che era stato preceduto dal Viaggio in America, si trova invece l'"Annunzio alli signori Associati alle opere del Visc. Chateaubriand» dove il Genio del Cristianesimo è definito l'opera principale dell'autore la cui pubblicazione è stata posticipata per poter prima «dar in luce alcune di quelle opere del Chateaubriand che di fresco furono in Francia pubblicate, e che fino a questo punto erano all'Italia straniere» (AA.VV. 1827-30, 10: 191); ancora una

${ }^{12}$ Alla fine di questo volume si trova anche l'elenco degli associati dell'edizione. 
volta, com'è evidente, si sottolinea l'alternanza tra l'inedito e il ben noto al fine di fidelizzare i propri lettori. D'altro canto, non ci si lascia atterrire dalle numerose traduzioni già esistenti del Génie, evidenziando le novità; non solo la nuova edizione parigina contiene una nuova prefazione «che daremo nel primo volume per intero tradotta», si sottolinea inoltre «che non mai edizione tanto diligente e compiuta del Genio del Cristianesimo sin qui s'è fatta, quanto si è quella che da noi si apparecchia»: nuove note, giudizi e opinioni francesi, i testi che l'autore cita per avvalorare le sue tesi. I lettori sono quindi invitati ad acquistare la nuova edizione in quanto essa è «la più compiuta di quante vennero fuori fino a quest'ora» (AA.VV. 1827-30, 10: 192). La volontà di rimarcare gli aspetti innovativi dell'ultima versione sul mercato non necessitano ulteriori sottolineature.

Dopo cinque volumi contenenti I Martiri, l'ultimo tomo della raccolta, il ventiquattresimo, propone la Lettera sopra l'arte del disegno nei paesaggi, seguita da Pensieri, Riflessioni e Massime, Poemi di Ossian, Milton e Davenant, Quadri della natura, Componimenti diversi. Interessante notare come i Poemi di Ossian siano preceduti dalla prefazione in cui Chateaubriand nomina i tre testi che ha tradotto da Smith-Dargo, Dutona e Gaulo-e come la nota editoriale specifichi che quest'ultimo sia stato omesso dal volume in quanto considerato «abbastanza noto all'Italia» (AA.VV. 1827-30, 24: 35): ancora una volta non ci si limita a pubblicare i testi così come editi in Francia, li si sceglie in base al pubblico della penisola rimarcando una linea editoriale precisa che non accoglie acriticamente quanto proviene da oltralpe, ma presenta un prodotto adattato al contesto e quindi verosimilmente più gradito al proprio lettorato.

L'impresa veneziana pare a questo punto passare il testimone a Firenze dove, dal 1831, «a spese di una società» e per i torchi di Simone Birindelli, inizia la pubblicazione di Opere di F.A. de Chateaubriand ${ }^{13}$ che in parte propone opere già contenute nella raccolta precedente, in parte presenta testi diversi. Vengono immesse nel mercato due versioni, una in $8^{\circ}$ e una in $4^{\circ}$, come segnala la Gazzetta di Firenze dell' 8 gennaio 1831 dove si specifica che, a seguito dello straordinario successo francese del testo I tre [sic!] Stuardi, la società che si propone di pubblicare l'intera opera dello scrittore ha già commissionato la traduzione di tale saggio ${ }^{14}$, destinato a seguire

${ }^{13}$ Fino al 1815 l'autore si firmava effettivamente François-Auguste e anche nella Notice bio-bibliografica posta nell'ultimo tomo dell'edizione Ladvocat (uscita proprio nel 1831) risulta ancora questo nome. Nella maggior parte dei frontespizi appare semplicemente come Chateaubriand, o tuttalpiù con il titolo di Visconte.

${ }^{14}$ L'opera verrà tradotta dal fiorentino Carlo Graziani che si occuperà anche della versione degli Studi o discorsi storici pubblicata per lo stesso Birindelli l'anno successivo. 
l'Itinerario di cui si annuncia la prossima uscita. La raccolta di Firenze è preceduta da un avviso degli editori che vale la pena riportare per intero:

Quando dapprima comparvero alla pubblica luce, e con ripetute edizioni si diffusero alcune opere del chiarissimo Visconte di Chateaubriand, suscitossi in chiunque le conobbe un vivo desiderio di tutte gustare le produzioni dell'esimio Scrittore. Ciò non pertanto niuno erasi per anche rivolto a sodisfar questo voto. Doveva naturalmente recar meraviglia siffatta trascuranza in un secolo, in cui tanto si favoriscono i buoni studii, pei quali d'ogni parte si da opera onde la lettura dei sommi riesca universalmente fruttuosa; in un secolo in cui, revocate dall'ingiusta oblivione le opere di quei grandi che o per forbito stile o per utili veri si distinsero, tutta è rivolta l'attenzione degli eruditi a promuover con queste la cultura della presente generazione. Che se ragion vuole diasi il primato a quelli scritti, sui quali vedasi impresso il venerabile suggello del tempo, vituperevole nondimeno fora per i presenti lasciare nell'oscurità un scrittore altamente benemerito della Famiglia Europea al quale per esser nel novero dei grandi non manca che il suffragio della posterità. E tanto più vituperevole fora per l'Italia nostra, ove le lettere e le arti della Grecia fuggitive trovaron ricovero e regno, e d'onde dopo il cieco tenebror della settentrionale barbarie prima emerse la face che di benefica luce rifulse poi per tutta Europa. Se questa giustizia era dunque dovuta al primo dei Francesi Scrittori viventi, a miglior dritto non poteva denegarsi al difensore magnanimo di quelle massime auguste che formarono per tanto volger di secoli la felicità delle incivilite nazioni.

Queste furono le considerazioni dalle quali veniva animata la Società Editrice quando intitolò nei suoi manifesti d'associazione al culto Pubblico Italiano l'intiera Collezione delle opere di Chateaubriand. Né la distolse il riflettere l'essere elleno parto di penna straniera. Avvegnachè, cessate le frivole gare, unironsi finalmente i popoli in bel ricambio di lumi e di cognizioni, e si spogliaron di quell'egoismo nazionale che fu mai sempre pregiudicevole al nobile scopo della universale cultura.

Non è delle nostre parti l'entrare nella profonda disamina delle produzioni che or si fanno di pubblica ragione, notandone con giusta critica i pregi o rilevandone i difetti, da cui niun grande andò immune giammai, se perfino alcuna volta

\section{...magnus dormitat Homerus.}

Il farlo sarebbe per certo commettersi ad un pelago non praticabile da picciolo legno. Si può non pertanto asserire che nel lodato Scrittore trovansi unite le due qualità, in che il Venosino fa consistere il sommo pregio d'ogni arte, l'utilità cioè mista al diletto (Gli editori 1831: n.p. [v-vi]).

A partire dalla volontà di assecondare i desideri del pubblico e di colmare una disdicevole lacuna, si dichiara che Chateaubriand - «scrittore altamente benemerito della Famiglia Europea» e «primo dei Francesi Scrittori viventi» - ha diritto a una «intiera Collezione» - che in realtà è

La raccolta prosegue col Genio del Cristianesimo nella versione di Luigi Toccagni che uscirà tra il 1833 e il 1835. 
ben lontana dall'essere completa - nonostante la sua «penna straniera», collocando l'impresa in un'ottica favorevole a una cultura universale scevra da egoismi nazionali. Sono in effetti innumerevoli le espressioni che suggeriscono comunione e superamento di confini e differenze, a partire da «universalmente fruttuosa» passando per «unironsi finalmente i popoli in bel ricambio di lumi e di cognizioni». Il progetto mira a unire la conoscenza dei classici a quella dei contemporanei, quella «presente generazione» che avrà in futuro il «suffragio della posterità»: le allusioni alla celeberrima polemica tra antichi e moderni vengono rese esplicite definendo l'Italia ricovero delle «lettere e le arti fuggitive della Grecia» la cui luce si è potuta propagare in tutta Europa «dopo il cieco tenebror della settentrionale barbarie». Chateaubriand merita di essere diffuso nella penisola in quanto «difensore magnanimo» del classicismo, mentre gli editori dichiarano di rifiutare una disamina di pregi e difetti delle sue opere ponendo come esempio Omero via una locuzione tratta da Orazio. Si tratta chiaramente di un testo politicamente e culturalmente schierato, avverso ai nazionalismi e alle opposizioni, ma deciso nel rimarcare il peso dei classici; un testo, come è evidente, privo di qualsiasi riferimento preciso alla figura dell'autore e delle sue opere e volto a sottolineare l'apertura dell'Italia alla letteratura straniera moderna, ma in un'ottica profondamente diversa da quella suggerita da Madame de Staël cinque anni prima.

Non entro nel dettaglio di questa raccolta e delle sue vicende editoriali, terminando sulla segnalazione che, salvo errore da parte mia, è in questi volumi che la produzione poetica di Chateaubriand viene presentata per la prima volta in Italia accompagnata dal testo originale ${ }^{15}$. Come si legge nella Gazzetta di Firenze di dicembre 1831, sono usciti per Birindelli «I Quadri della Natura, e diverse altre Poesie alle versioni delle quali si è creduto ben fatto porre il testo a fronte per maggiore soddisfacimento dei Lettori di queste interessanti Opere» (Avvisi 1831: 4). Vi si potrebbe vedere una sorta di omaggio a colui che aveva pubblicato il suo Paradis perdu ponendo a piè di pagina l'originale di Milton, a riprova della lotta intrapresa col testo, al fine di proporre una versione letterale e al contempo curata al lettore, entità che il traduttore deve rispettare giacché «si vous ne vous souciez guère de lui, il se souciera encore moins de vous» (Chateaubriand 2012: 89).

${ }^{15}$ Vale la pena ricordare, sia pure a mero livello di suggestione, come nel 1806 la Risposta alla lettera del signor di Chateaubriand sopra Venezia di Giustina Renier Michiel venga pubblicata dopo il testo di Chateaubriand, datato 30 luglio 1806 e pubblicato nel Mercure de France, che si apre con l'affermazione "A Venise on venoit de publier une nouvelle traduction du Génie du Christianisme». I due testi francesi sono affiancati dalle loro traduzioni e quella della missiva della Veneziana è dichiarata essere a opera dell'abate Saverio Bettinelli. 
Appendice: le raccolte

\begin{tabular}{|c|c|c|}
\hline Paris: Ladvocat (1826-1831) & $\begin{array}{l}\text { Venezia: Girolamo Tasso (1827- } \\
1830)\end{array}$ & $\begin{array}{l}\text { Firenze: [Simone Brindelli] } \\
\text { (1831-1833) }\end{array}$ \\
\hline $\begin{array}{l}\text { I-II (1826) } \\
\text { Essai sur les Révolutions } \\
\text { III (1827) } \\
\text { Mélanges historiques } \\
\text { IV-Vter (1831) } \\
\text { Etudes ou discours historiques } \\
\text { VI-VII (1827) } \\
\text { Voyages en Amérique et en } \\
\text { Italie } \\
\text { VIII-X (1826) } \\
\text { Itinéraire de Paris à Jérusalem } \\
\text { XI-XV (1827) } \\
\text { Génie du Christianisme } \\
\text { XVI (1826) } \\
\text { Atala, René et les Abencérages } \\
\text { XVII-XVIIIbis (1826-1827) } \\
\text { Les Martyrs } \\
\text { XIX-XX (1826) } \\
\text { Les Natchez } \\
\text { XXI (1826) } \\
\text { Mélanges littéraires } \\
\text { XXII (1828) } \\
\text { Mélanges et poésies } \\
\text { XXIII (1827-1828) } \\
\text { Mélanges politiques } \\
\text { XXIV-XXV (1827-1828) } \\
\text { Mélanges politiques } \\
\text { XXVI (1827) } \\
\text { Polémique } \\
\text { XXVII (1828) } \\
\text { De la liberté de la presse } \\
\text { XXVIII (1831) } \\
\text { Table avec une notice sur la } \\
\text { vie et les ouvrages de l'auteur }\end{array}$ & $\begin{array}{l}\text { I (1827) } \\
\text { Avventure dell'ultimo Abencerragio } \\
\text { II-III (1827) } \\
\text { I Natchi, poema } \\
\text { IV-V (1828) } \\
\text { I Natchi, continuazione } \\
\text { VI (1827) } \\
\text { Renato, Atala } \\
\text { VII-X (1828) } \\
\text { Viaggi in America ed in Italia } \\
\text { XI-XVIII (1829) } \\
\text { Genio del Cristianesimo } \\
\text { XIX-XXIII (1829-1830) } \\
\text { I Martiri } \\
\text { XXIV (1830) } \\
\text { Lettera sopra l'arte del disegno nei } \\
\text { paesaggi }\end{array}$ & $\begin{array}{l}\text { I (1831) } \\
\text { Itinerario da Parigi a Geru- } \\
\text { salemme } \\
\text { II (1831) } \\
\text { I quattro Stuardi; Pensieri, } \\
\text { riflessioni, massime; Poesie, } \\
\text { Quadri della Natura, Poemi } \\
\text { diversi; Avventure dell'ultimo } \\
\text { Abencerragio; Considerazioni } \\
\text { su l'Inghilterra e gli inglesi; I } \\
\text { Martiri; Considerazioni sopra } \\
\text { Young, Considerazioni sopra } \\
\text { Shakespeare } \\
\text { III-IV (1832) } \\
\text { Studi o discorsi storici } \\
\text { V-VII (1833) } \\
\text { Genio del Cristianesimo }\end{array}$ \\
\hline
\end{tabular}

\section{BIBLIOGRAFIA}

AA.VV. (1827-1830). Paratesti degli editori e dei traduttori. In Opere varie del visconte Chateaubriand recate in italiano, 24 vol. Venezia: Girolamo Tasso.

Avvisi (1831, 6 dicembre). Gazzetta di Firenze, 146, 4.

Berchet, J.-C. (2012). Chateaubriand. Paris: Gallimard «Biographies $\mathrm{NRF}$ ».

Berman, A. (1995). Pour une critique des traductions: John Donne. Paris: Gallimard. 
Bolza, G. B. (1840). Edizioni illustrate, di opere italiane originale e tradotte, in «Rassegna bibliografica». Rivista Viennese, III, 282-291.

Borgese, G. A. (1930). Postfazione. In Stendhal, La certosa di Parma (pp. 671-692). Milano: Mondadori.

Bougeard-Vetö, M.-E. (2005). Chateaubriand traducteur. De l'exil au Paradis perdu. Paris: Champion.

Chateaubriand, F.-R. (2003-2004). Mémoires d'outre-tombe, a cura di J.-C. Berchet, 2 vol. Paris: Bordas.

Chateaubriand, F.-R. (2012). Essai sur la littérature anglaise et considérations sur le génie des hommes, des temps et des révolutions, a cura di S. Baudoin. Paris: Société des textes français modernes.

Cordié, C. (1969). Traduzioni e edizioni italiane di scritti di Chateaubriand dal 1801 a oggi. In Chateaubriand e l'Italia (pp. 49-66). Roma: Accademia Nazionale dei Lincei.

Fedelini, E. (1932). Una poetessa veneta del primo 800: Edvige de Battisti di San Giorgio de Solari. Verona: Tipografia Operaia.

Gli editori (1831). In Itinerario da Parigi a Gerusalemme e da Gerusalemme a Parigi andando per la Grecia e ritornando per l'Egitto, la Barberia e la Spagna di F.-R. de Chateaubriand. Traduzione dall'originale francese di F. G. Firenze: Simone Brindelli.

Guerra, A. (2004). Il vile satellite del trono. Lorenzo Ignazion Thjulen: un gesuita svedese per la controrivoluzione. Milano: Franco Angeli.

Maffei, A. \& gli editori (1857). Paratesti. In Il Paradiso Perduto. Poema di Giovanni Milton, traduzione del Cavaliere Andrea Maffei (pp. xiii-xiv e v-vii). Torino: Unione tipografico editrice.

Rabizzani, G. (1918). Chateaubriand nel Risorgimento italiano. Rivista d'Italia, XXI, 273-294.

Renier Michiel, G. (1806). Risposta alla lettera del signor di Chateaubriand sopra Venezia. Venezia: Tipografia Fracasso.

Riberette, P. (1965-1966). Chateaubriand et le libraire Piatti. Bulletin de la Société Chateaubriand, 9, 36-39.

Rosi, I. (2015). Letture delle Memorie (1834-1994). In Chateaubriand, Memorie d'oltre-tomba, Introduzione di C. Garboli, Nuova edizione a cura di I. Rosi e F. Vasarri, Traduzione di I. Rosi, F. Martellucci e F. Vasarri (Vol. 1, pp. clx-clxv), 2 vol. Milano: Einaudi.

Sullam Calimani, A. V. (1998). Esotismi e Neologismi nelle traduzioni di Atala. Lingua Nostra, 59, 84-89.

Torop, P. (2009). La traduzione totale. Tipi di processo traduttivo nella cultura, edizione italiana a cura di B. Osimo. Milano: Hoepli.

Toury, G. (1980). In Search of a Theory of Translation. Tel Aviv: Porter Institute for Poetics and Semiotics. 
Toury, G. (1995). Descriptive Translation Studies and Beyond. Amsterdam/ Philadelphia: John Benjamins Publishing.

Vasarri, F. (2015). Letture delle Memoria (1995-2015). In Chateaubriand, Memorie d'oltre-tomba, Introduzione di C. Garboli, Nuova edizione a cura di I. Rosi e F. Vasarri, Traduzione di I. Rosi, F. Martellucci e F. Vasarri (Vol. 1, pp. clxvii-clxxviii), 2 vol. Milano: Einaudi.

Wilhelm, J. E. (2004). La traduction, principe de perfectibilité, chez Mme de Staël. Meta, 493, 692-705.

\section{MR CHATEAUBRIAND TRANSPORTED INTO ITALIAN. $19^{\mathrm{TH}}$ CENTURY MEDIATIONS}

\section{Summary}

On the basis of the characteristics that would allow the creation of an annotated repertoire of the Italian translations of Chateaubriand, and starting from the presentation of some significant cases, the article aims to underline that the study of $19^{\text {th }}$ century mediation requires a trans-disciplinary approach. The analysis of two early $19^{\text {th }}$ century collections of Chateaubriand's oeuvre leads to considerations on the transmission of French culture into the Italian context.

Keywords: Chateaubriand, History of Mediation, Practice and Theory of Translation, Italian Reception of French Literature, $19^{\text {th }}$ Century Culture, trans-disciplinary Studies. 